\title{
Eine Frage der Rechte und Pflichten
}

\section{Die Zahl von Publikationen zu öko-effizienten Dienstleistungen wächst schnell. Eine vergleichbare reale Entwicklung solcher Konzepte im Konsum- güterbereich steht hingegen noch aus. Von welchen Faktoren hängt die noch geringe Konsumentenakzeptanz ab? Mit der Erweiterung des Verfügungs- rechteansatzes der Property-Rights-Theorie wird in diesem Beitrag eine neve Struktur relevanter Einflussfaktoren vorgestellt und für die Bedürfnis- felder Automobilität und saubere Wäsche empirisch getestet (1).}

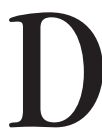

Von Ulf Schrader ie Hintergrundphilosophie öko-effizienter Dienstleistungen beruht auf der Überlegung, dass Menschen letztlich keine Produkte kaufen wollen, sondern ihre Bedürfnisse befriedigen. Sie sind nicht am Kauf von Auto, Waschmaschine und Elektrobohrer interessiert, sondern an individueller Fortbewegung, sauberer Wäsche und dem Loch in der Wand (2). Diese sogenannte Funktionsorientierung hat den Blick geöffnet für Alternativen zum ressourcenintensiven Konsum von Produkteigentum, sie birgt jedoch auch die Gefahr, die Bedeutung des Produkteigentums auf nur eine Funktion zu verengen.

Materielles Produkteigentum erfüllt für Konsumenten in aller Regel ein ganzes Bündel an Bedürfnissen. Dieser Aspekt ist besonders relevant im Hinblick auf die Akzeptanz der Ersetzung von Konsumgütereigentum durch öko- effiziente Nutzungs- und Ergebnisdienstleistungen (3). Wer sich beispielsweise gegen das Car-Sharing und für den Privatwagen entscheidet, tut dies in der Regel nicht, weil er glaubt, mit dem eigenen Auto besser von A nach B zu kommen. Konsumgütereigentum besitzt für Konsumenten vielfach eine symbolische Bedeutung als materialisierter Ausdruck der wahrgenommenen oder angestrebten Identität; diese Symbolbedeutung stellt ein zentrales Hemmnis für die Akzeptanz eigentumsersetzender Dienstleistungen dar (4). Aber selbst wenn man Produkte als bloße „Dienstleistungserfuillungsmaschinen" (Schmidt-Bleek) betrachtet, sind mehr Aspekte relevant als die reine Nutzungsmöglichkeit.

Einen Ansatz, die Vielfalt dieser funktionalen Aspekte zu systematisieren und gleichzeitig Eigen- tum und eigentumsersetzende Dienstleistungen vergleichbar zu machen, liefert der Verfügungsrechteansatz der Property-Rights-Theorie, der Eigentum in bis zu fünf Verfügungsrechte ausdifferenziert (5). Diesen Rechten lassen sich nun nahezu spiegelbildlich auch Verfügungspflichten gegenüberstellen (vgl. Tabelle 1).

\begin{tabular}{|l|l|}
\hline \multicolumn{2}{|c|}{ Tabelle 1: Verfügungsrechte und -pflichten } \\
\hline Verfügungsrechte & Verfügungspflichten \\
\hline \hline Nutzungsrecht & Nutzungspflicht \\
\hline Ausschlussrecht & Unterbringungspflicht \\
\hline Veränderungsrecht & \begin{tabular}{l} 
Instandhaltungspflicht \\
\hline Veräußerungsrecht
\end{tabular} \\
\hline Veräußerungs- bzw. \\
\hline Gewinnaneignungsrecht & $\begin{array}{l}\text { Entsorgungspflicht } \\
\text { Kostenübernahmepflicht } \\
\text { Quelle: eigene Darstellung }\end{array}$ \\
\hline
\end{tabular}

\section{Verfügungsrechte und -pflichten}

Während einem Eigentümer alle Verfügungsrechte gleichzeitig zugeordnet sind, kommt es für den Konsumenten eigentumsersetzender Dienstleistungen hier überwiegend zu Einschränkungen. Zwar sind das Nutzungsrecht bei Nutzungsdienstleistungen sowie generell die Möglichkeit zur Bedürfnisbefriedigung durch Gütereinsatz, also das Gewinnaneignungsrecht im weiteren Sinne, weiterhin gegeben, hinsichtlich der anderen Rechte erfolgt jedoch eine Reduktion bis hin zum vollständigen Wegfall. Diesem Nachteil kann der Vorteil einer Ausweitung der Nutzungs- und Gewinnaneignungsrechte gegenüberstehen: Der Konsument von Nutzungsdienstleistungen hat in der Regel die Möglichkeit, nicht nur ein Produkt, sondern eine gesamte Produktflotte zu nutzen. Der Nutzer einer Ergebnisdienstleistung kann seine Bedürfnisbe- friedigungsmöglichkeiten und damit sein Gewinnaneignungsrecht durch Ergebnisse erweitern, die im Vergleich zur Eigentumsnutzung besser oder vielfältiger sind.

Interessant wird die Inanspruchnahme eigentumsersetzender Dienstleistungen aber vor allem dann, wenn auch die Verfügungspflichten berücksichtigt werden. Diese Pflichten des Eigentums, die von Eigentumstheoretikern bisher weitgehend vernachlässigt wurden, lassen sich wie folgt charakterisieren:

Nutzungspflicht: Der Konsument hat nicht nur das Recht, sein Eigentum zu nutzen; will er die angestrebte Bedürfnisbefriedigung erreichen, ist er - sofern es sich nicht um ein reines Kontemplationsobjekt handelt - auch zur Nutzung verpflichtet. Denn der Produktnutzen wird nicht bereits mit dem Kauf des Produktes erworben, sondern muss vom Konsumenten als Co-Produzenten erst selbst erzeugt werden.

- Unterbringungspflicht: Der Konsument hat nicht nur das Recht, sein Eigentum vor anderen zu verschließen, er hat auch die Pflicht, es so unterzubringen, dass es Dritten nicht zur Last fällt.

- Instandhaltungspflicht: Der Konsument hat nicht nur das Recht zur Abnutzung und Zerstörung seines Eigentums, er hat auch die Pflicht, es zumindest soweit Instand zu halten, dass es Andere nicht gefährdet.

- Veräußerungs- bzw. Entsorgungspflicht: Der Konsument hat nicht nur das Recht zur Veräußerung seines Eigentums, er hat auch die Pflicht zu einer geregelten Übertragung der Verfügungsrechte an Andere bzw. zur rechtmäßigen Entsorgung, wenn er sich seines Eigentums entledigen will oder muss.

- Kostenübernahmepflicht: Der Konsument hat nicht nur das Recht zur Aneignung des mit seinem Eigentum erzeugten Gewinnes, er hat auch die Pflicht zur Übernahme der Kosten, die mit Aneignung und Ausübung der verschiedenen Verfügungsrechte und -pflichten verbunden sind. $\mathrm{Zu}$ diesen Kosten zählen auch nicht-monetäre Kosten in Form von Zeit und Mühe.

Während der Eigentümer all diese Pflichten allein zu tragen hat, kann der Nutzer eigentumsersetzender Dienstleistungen einen Großteil der Verfügungspflichten an den Anbieter ,outsourcen“. Allerdings sind eigentumsersetzende Dienstleistungen meistens mit erhöhten Personalkosten auf Anbieterseite, die auf den Konsumenten umgelegt werden, und zusätzlichen nicht-monetären Transaktionskosten auf der Konsumentenseite verbunden. 


\section{- Empirische Überpröfung}

Die Ausgangshypothese der empirischen Überprüfung war nun, dass die veränderte Verteilung der Verfügungsrechte und -pflichten für Konsumenten Vor- und Nachteile mit sich bringt, deren Wahrnehmung in einem signifikanten Zusammenhang mit der Akzeptanz eigentumsersetzender Dienstleistungen steht. Akzeptanz wurde dabei definiert als positive Einstellung mit entsprechender Handlungsintention. Sie stellt somit eine Vorstufe der tatsächlichen Inanspruchnahme der Dienstleistung dar. Die Untersuchung erfolgte anhand des viel diskutierten Beispiels ,Substitution des eigenen Autos durch die Nutzungsdienstleistung Car-Sharing" sowie des fiktiven Angebotes „Ersetzung der eigenen Waschmaschine durch die Ergebnisdienstleistung Wasch-Service (inkl. Hol- und Bringdienst)“. Die Daten basieren auf zwei parallelen Haushaltsbefragungen, wobei die Stichprobe das Ergebnis einer geschichteten $\mathrm{Zu}$ fallsauswahl unter den erwachsenen Bewohnern der Stadt Hannover darstellt. Tabelle 2 fasst die zentralen Untersuchungsergebnisse zusammen (6).

Berücksichtigt wurden hier nur Verfügungsrechte und -pflichten, die für den Konsumenten grundsätzlich Relevanz besitzen. Nach diesen Ergebnissen ist die Car-Sharing-Akzeptanz um so höher, je positiver

die Erweiterung des Nutzungsrechtes durch die Möglichkeit zur Flotten- und Quernutzung, der Wegfall der Unterbringungspflicht aufgrund vorhandener Stellplätze,

die Einschränkung der Instandhaltungspflicht durch Aktivitäten des Anbieters und

die Einschränkung der Fixkostenübernahmepflicht durch Verteilung auf alle Nutzer wahrgenommen werden. Die Car-Sharing-Akzeptanz fällt um so geringer aus, je negativer die Einschränkung von Ausschluss- und Veränderungsrecht sowie die Erweiterung der Übernahmepflicht für nicht-monetäre Transaktionskosten (zum Beispiel durch den Weg zum Stellplatz oder die aufwändigere Abrechnung) eingeschätzt werden. Der Aspekt der Erweiterung der Übernahmepflicht für monetäre Kosten erbrachte hier kein signifikantes Ergebnis, vermutlich weil der sonst zentrale Aspekt zusätzlicher Personalkosten aufgrund der zum Erhebungszeitpunkt (Januar 1998) noch überwiegend ehrenamtlichen Organisation des Car-Sharing nicht berücksichtigt wurde.

Beim Wasch-Service erwies sich das durch den entgeltlichen Bügelservice erweiterte Gewinnaneignungsrecht als akzeptanzfördernd. Gleiches gilt für die entfallende Nutzungs- und Unterbringungspflicht sowie die eingeschränkte Pflicht zur Fixkostenübernahme. Dem stehen zwei Faktoren gegenüber, welche die Akzeptanz negativ beeinflussten: der Wegfall des Ausschlussrechtes und der damit einhergehende Kontakt der eigenen Wäsche mit fremden Wäschestïcken und dem Servicepersonal sowie die

\begin{tabular}{|c|c|c|}
\hline Konsumentenseitige Verfügungsrechte und -pflichten & \multicolumn{2}{|c|}{ Korrelation mit der Konsumentenakzep- } \\
\hline \multicolumn{3}{|l|}{$\operatorname{tanz}$} \\
\hline \multicolumn{3}{|l|}{ im Vergleich zum Eigentum } \\
\hline & Car-Sharing & Wasch-Service \\
\hline & $N=186$ & $N=180$ \\
\hline \multicolumn{3}{|l|}{ Vorteile } \\
\hline Erweiterung des Nutzungsrechtes & $0,25^{\star \star}$ & - \\
\hline Erweiterung des Gewinnaneignungsrechtes & - & $0,18^{*}$ \\
\hline Wegfall der Nutzungspflicht & - & $0,32^{\star \star}$ \\
\hline Wegfall der Unterbringungspflicht & $0,22^{\star \star}$ & $0,17^{\star}$ \\
\hline Einschränkung der Instandhaltungspflicht & $0,15^{\star}$ & - \\
\hline Einschränkung der Übernahmepflicht für Fixkosten & $0,26^{\star \star}$ & $0,31^{\star \star}$ \\
\hline \multicolumn{3}{|l|}{ Nachteile } \\
\hline Einschränkung des Ausschlussrechtes & $-0,22^{\star \star}$ & $-0,27^{\star \star}$ \\
\hline Einschränkung des Veränderungsrechtes & $-0,23^{\star \star}$ & - \\
\hline Erweiterung der Übernahmepflicht für monetäre Kosten & $-0,05 n \cdot s$. & $-0,23^{\star \star}$ \\
\hline Erweiterung der Übernahmepflicht für nicht-monetäre Transaktionskosten & $-0,19 *$ & $-0,06$ n.s. \\
\hline \multicolumn{3}{|l|}{ Signifikanzniveau: ${ }^{* \star}<0,01 ;{ }^{*}<0,05 ;$ n.s. $=$ nicht signifikant } \\
\hline — = keine sinnvolle Korrelation möglich & & \\
\hline
\end{tabular}

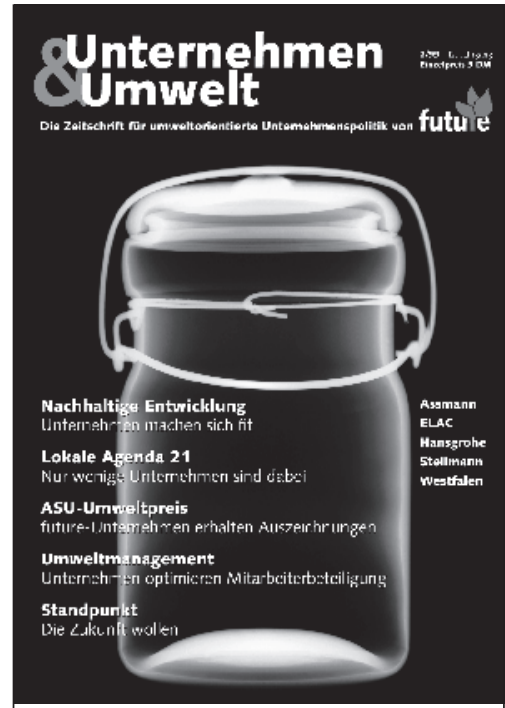

Unternehmen \& Umwelt

- ist die Zeitschrift für umweltorientierte Unternehmenspolitik von future e.V.,

- berichtet über den neuesten Stand zum betrieblichen Umweltmanagement in Theorie und Praxis,

- bietet vier Mal im Jahr ein Schwerpunktthema,

Unternehmensbeispiele, Literaturund Veranstaltungshinweise und aktuelle future-Nachrichten.

Zum Beispiel Heft 2/99:

\section{Nachhaltigkeit}

- Nachhaltige Entwicklung: Unternehmen machen sich fit.

- Lokale Agenda 21: Nur wenige Unternehmen sind dabei.

- Betrieblicher Umweltschutz: Assman, ELAC, Hansgrohe, Steilmann.

- Umweltmanagement: Unternehmen optimieren die Mitarbeiterbeteiligung.

Fordern Sie Ihr kostenloses

Probeexemplar an:

Fon: 0234 - 97995 - 13 Fax: $0234-97995-14$

future e.V. Büro Bochum Am Varenholt 123 44797 Bochum email: future@umis.de 

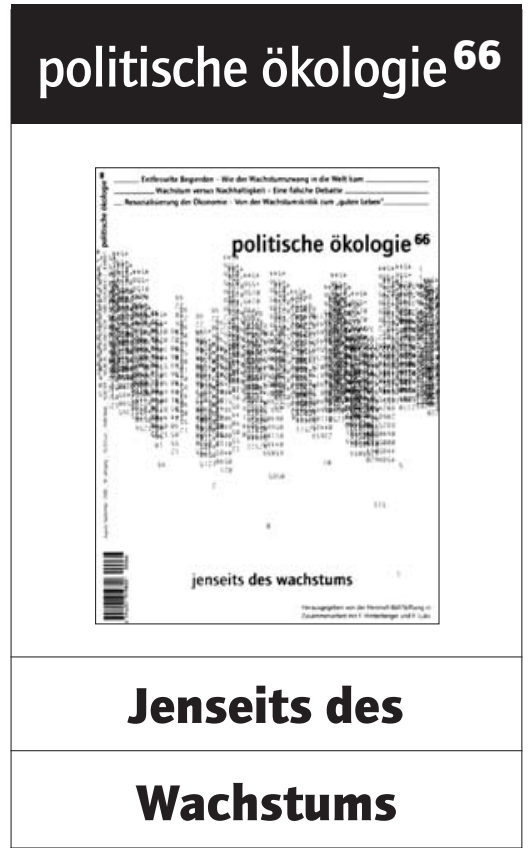

Deutschland im Jahr 2000: Wandel und Reformen werden allenthalben angemahnt, der Umbau von Industriegesellschaft und Sozialstaat beschworen, der Aufbruch in Internet-, Biotechund sonstige Zeitalter gefeiert. Wenn von Wandel die Rede ist, heißt das im Wesentlichen: Weiter so - nur besser, schneller und effizienter. Der Hinweis auf „Grenzen des Wachstums“, nun bereits fast 30 Jahre alt, scheint dagegen nicht mehr relevant zu sein.

Die politische ökologie 66 bringt das Thema Wachstum wieder zurïck auf die Tagesordnung. Sie versammelt prominente internationale Stimmen, die mahnen, dass das Problem nicht nur nicht gelöst ist, sondern immer drängender wird. So vielfältig wie die Diagnosen sind auch die Therapievorschläge. Das Heft gibt einen umfassenden Überblick über den Stand der Wachstumsdiskussion und zeigen, wie aktuell diese Frage nach wie vor ist. Wohin wachsen wir?

Mit Beiträgen von Fred Luks, Friedrich Hinterberger, Herman E. Daly, Gerhard Scherhorn, Elmar Altvater, Klaus Töpfer, Ernst Ulrich von Weizsäcker und anderen.

\section{www.oekom.de}

Jetzt für 19,80 Mark direkt

zu bestellen bei:

pan adress

politische ökologie Leserservice

Semmelweisstr. 8

D-82152 Planegg

Fon $++49|(0) 89| 85709-145$

Fax $++49|(0) 89| 85709-131$ erweiterte Übernahmepflicht durch personalbedingte monetäre Kosten. Hinsichtlich der Bewertung der nicht-monetären Transaktionskosten war kein signifikanter Zusammenhang festzustellen, was auf eine uneinheitliche Interpretation des Hol- und Bringdienstes zurückgeführt werden kann.

\section{Schlussfolgerungen}

Folgende Schlussfolgerungen lassen sich aus der durchgeführten Untersuchung ziehen:

- Der um Verfügungspflichten ergänzte Eigentumsbegriff der Verfügungsrechtetheorie ist grundsätzlich geeignet, die funktionale Bedeutung einer Substitution des Eigentums durch Dienstleistungen zu systematisieren. Die empirischen Ergebnisse zeigen die Relevanz der Wahrnehmung veränderter Verfügungsrechte und -pflichten für die Akzeptanz eigentumsersetzender Dienstleistungen.

- Um Konsumentenakzeptanz zu erreichen, ist die Unternehmenspraxis aufgefordert, die mit eigentumsersetzenden Dienstleistungen verbundenen Verfügungsrechte und -pflichten entsprechend zu gestalten und zu kommunizieren. Dabei gilt es, die Stärken - insbesondere die Erweiterung von Nutzungs- und Gewinnaneignungsrecht sowie die Einschränkung der Fixkostenübernahmepflicht - zu stärken und die Schwächen - insbesondere die Einschränkung von Ausschluss- und Veränderungsrecht und die Erweiterung von Personal- und Transaktionskosten - abzumildern.

Weiterhin bleibt jedoch das Problem bestehen, dass die Verfügungspflichten des Eigentums nur in wenigen Produktbereichen so hoch sind, dass Konsumenten überhaupt über einen Wechsel zu Dienstleistungen nachdenken. Will der Staat diesen Wechsel fördern, muss er die herrschenden Rahmenbedingungen durch entsprechende steuer- und ordnungsrechtliche Maßnahmen ändern. Damit ließen sich die mit Eigentum verbundenen Pflich-

\section{Auf dem Weg zum nach- baltigen Unternebmen?}

ist das Thema

des Informationsdienstes

Ökologisches Wirtschaften 1/01

\section{Wenn Sie potenzielle Beiträge haben, wenden Sie sich bitte an die Redaktion.}

ten, zum Beispiel die der Entsorgung und der Kostenübernahme, im Vergleich zum Konsum ohne Eigentum verschärfen.

\section{Anmerkungen}

(1) Die hier dargestellten Forschungsergebnisse stammen aus dem Projekt "Eigentumsloser Konsum - Untersuchung und exemplarische Umsetzung eines ökologischeren Konsumstils", das der Lehrstuhl Marketing 1: Markt und Konsum mit finanzieller Unterstützung der Deutschen Bundesstiftung Umwelt durchgeführt hat.

(2) Vgl. zu dieser Argumentation z.B. Leinkauf, S./ Zundel, S.: Funktionsorientierung und Ökoleasing - Strategien und Instrumente einer proaktiven Umweltpolitik, Schriftenreihe des IÖW, Nr. 79/94, Berlin 1994, S. $5 f f$.; Schmidt-Bleek, F.: Das MIPS-Konzept. Weniger Naturverbrauch - mehr Lebensqualität durch Faktor 10, München 1998, S. $63 \mathrm{ff}$.

(3) Zur Einteilung öko-effizienter Dienstleistungen in Produktdienstleistungen (z.B. Reparaturen), Nutzungsdienstleistungen (z.B. Vermietung) und Ergebnisdienstleistungen (z.B. Handwerkerdienste) vgl. z.B.

Bierter, W./ Stahel, W.R./ Schmidt-Bleek, F.: Öko-intelligente Produkte, Dienstleistungen und Arbeit, Wuppertal Spezial 2, Wuppertal 1996, S. 53ff.;

Schrader, U.: Consumer Acceptance of Eco-efficient Services. A German Perspective, in: Greener Management International, Vol. 25 (1999), S. 105-121, insb. $106 f f$. (4) Vgl. z.B. Scholl, G.U./ Hirschl, B./ Tibitanzl, F.: Produkte länger und intensiver nutzen. Zur Systematisierung und ökologischen Beurteilung alternativer Nutzungskonzepte, Schriftenreihe des IÖW 134/98, Berlin 1998, S. 53ff.; Belz, F.M.: Ökologische Innovationen in der Kreislaufwirtschaft: Leistungs- statt Produktverkauf, IWÖ-Diskussionsbeitrag Nr. 62, St. Gallen 1998, S. $29 f$.

(5) Vgl. z.B. Silver, M.: Foundations of Economic Justice, New York 1989, S. 17ff.; vgl. zur Diskussion von Verfügungsrechten im Kontext öko-effizienter Dienstleistungen insb. Hockerts, K.: Konzeptualisierung ökologischer Dienstleistungen. Dienstleistungskonzepte als Element einer wirtschaftsökologisch effizienten Bedürnnisbefriedigung, IWÖ Diskussionspapier Nr. 29, St. Gallen 1995, S. $18 f f$.

(6) Die Ermittlung des Akzeptanzurteils erfolgte hier im Rahmen einer Conjoint-Analyse, während die Bewertung der Verfügungsrechtestruktur über Multi-ltem-Skalen gemessen wurde; vgl. zu den methodischen Hintergründen sowie zu dem verwendeten Erhebungsinstrument Schrader, U.: Die Konsumentenakzeptanz eigentumsersetzender Dienstleistungen, Hannover (in Vorbereitung).

\section{Der Autor}

Ulf Schrader ist wissenschaftlicher Mitarbeiter am Lehrstuhl Marketing 1: Markt und Konsum der Universität Hannover.

Kontakt: Universität Hannover, Königsworther Platz 1, 30167 Hannover, Tel. 0511/762-5629, Fax-5630, E-mail: us@muk.ifb.uni-hannover.de 
(c) 20I0 Authors; licensee IÖW and oekom verlag. This is an article distributed under the terms of the Creative Commons Attribution Non-Commercial No Derivates License (http://creativecommons.org/licenses/by-nc-nd/3.o/), which permits unrestricted use, distribution, and reproduction in any medium, provided the original work is properly cited. 